\title{
Correction to: Ugandan Study Participants Experience Electronic Monitoring of Antiretroviral Therapy Adherence as Welcomed Pressure to Adhere
}

\author{
Jeffrey I. Campbell ${ }^{1} \cdot$ Nir Eyal $^{2} \cdot$ Angella Musiimenta $^{3} \cdot$ Bridget Burns $^{4} \cdot$ Sylvia Natukunda ${ }^{3} \cdot$ Nicholas Musinguzi $^{3}$. \\ Jessica E. Haberer ${ }^{4}$
}

Published online: 15 June 2020

(c) Springer Science+Business Media, LLC, part of Springer Nature 2020

\section{Correction to: AIDS and Behavior (2018) 22:3363-3372 https://doi.org/10.1007/s10461-018-2200-8}

The original version of this article unfortunately contained an error. The authors would like to correct the error with this erratum.

In the original publication of the article, the number of "EAM Users" with education level of "University/vocational" in Table is listed as 5 [12.5\%], where it should be listed as 3 [7.5\%]. The corrected Table 1 is listed below. The correction of this error does not impact the results, interpretation, or message as presented in the published paper.

The original article can be found online at https://doi.org/10.1007/ s10461-018-2200-8.

Jeffrey I. Campbell

Jeffrey.Campbell@bmc.org

1 Department of Pediatrics, Boston Medical Center, and Boston Children's Hospital, One Boston Medical Center Pl, Dowling 3rd Floor, Boston, MA 02118, USA

2 Harvard T.H. Chan School of Public Health, Boston, MA, USA

3 Mbarara University of Science and Technology, Mbarara, Uganda

4 Massachusetts General Hospital, Boston, MA, USA 
Table 1 Participant characteristics

\begin{tabular}{|c|c|c|c|}
\hline UARTO participants & EAM users $(n=40)$ & EAM non-users $(n=20)$ & $\begin{array}{l}\text { p-value (difference between } \\
\text { EAM users and non-users) }\end{array}$ \\
\hline Age (median [IQR]) & $41[35-46]$ & $45[42-50]$ & 0.03 \\
\hline Female (\%) & 70 & 65 & 0.77 \\
\hline Literate $(\%)$ & 90 & 85 & 0.68 \\
\hline Education level (n [\%]) & & & 0.77 \\
\hline Never attended school & $4[10]$ & $2[10]$ & \\
\hline Primary & $23[57.5]$ & $11[55]$ & \\
\hline O-Level ${ }^{\mathrm{a}}$ & $9[22.5]$ & $3[15]$ & \\
\hline A-Level ${ }^{\mathrm{a}}$ & $1[2.5]$ & $3[15]$ & \\
\hline University/vocational & $3[7.5]$ & $1[5]$ & \\
\hline Post-graduate & $0[0]$ & $0[0]$ & \\
\hline \multicolumn{4}{|l|}{ Socioeconomic status } \\
\hline Earns a salary? (n [\%]) & $8[20 \%]$ & $5[25 \%]$ & 0.74 \\
\hline Monthly salary (median [IQR]) $(\mathrm{USD})^{\mathrm{b}}$ & $\$ 65[\$ 44-\$ 130]$ & $\$ 116[\$ 87-\$ 116]$ & 0.42 \\
\hline Monthly non-salaried income (median [IQR]) $(\mathrm{USD})^{\mathrm{b}}$ & $\$ 12[\$ 4-\$ 36]$ & $\$ 63[\$ 24-\$ 81]$ & 0.04 \\
\hline Monthly household expenditures (median [IQR]) (USD) ${ }^{\mathrm{b}}$ & $\$ 75[\$ 39-\$ 148]$ & $\$ 87[\$ 22-\$ 171]$ & 0.67 \\
\hline Time from UARTO enrollment to interview (years, mean [SD]) & $5.5[2.7]$ & $8.0[1.1]$ & $<0.0001$ \\
\hline UARTO RA’s & $\mathrm{n}=6$ & & \\
\hline Age (median [IQR]) & $36[34-38]$ & & \\
\hline Female (n [\%]) & $4[66]$ & & \\
\hline
\end{tabular}

UARTO Uganda AIDS Rural Treatment Outcomes study, EAM electronic adherence monitor, IQR interquartile range, USD United States Dollar, $S D$ standard deviation, $C A B$ Community Advisory Board, REC Research Ethics Committee

${ }^{a}$ In the Ugandan education system, O-Level indicates completion of secondary school. A-Level is two-year, post-secondary, pre-university schooling

${ }^{\mathrm{b}}$ Monetary conversion calculated at 1 US Dollar=3445 Ugandan Shillings (as of January 12, 2015)

Publisher's Note Springer Nature remains neutral with regard to jurisdictional claims in published maps and institutional affiliations. 